\title{
A Rapid and Simple Method for Brassica Napus Floral-Dip Transformation and Selection of Transgenic Plantlets
}

\author{
Juan Li \\ Institute of Life Sciences, Jiangsu University \\ Zhenjiang 212013, China \\ E-mail: lijuan151961591@163.com \\ Xiaoli Tan (Corresponding author) \\ Institute of Life Sciences, Jiangsu University \\ Zhenjiang 212013, China \\ Tel: 86-511-8291-4326 E-mail: xltan@ujs.edu.cn
}

Fuge Zhu

Institute of Life Sciences, Jiangsu University

Zhenjiang 212013, China

E-mail: zfg12@163.com

Jinging Guo

School of Food \& Biological Engineering, Jiangsu University

Zhenjiang 212013, China

E-mail: 798572914@qq.com

The research is financed by the National Basic Research Program (973 Program) of China (No.2006CB101600), Talent Fund of Jiangsu University (05JDG003) and Student Research Project of Jiangsu University (08A088).

\begin{abstract}
The floral-dip method of transformation by immersion of inflorescences in a suspension of Agrobacterium was applied in the Brassica napus transformation, but it involves a number of relatively time-consuming and laborious steps, including manipulating an Agrobacterium tumefaciens culture and aseptic procedures for the selection of plant lines harboring antibiotic-selection markers. It calls for a long time to prepare the buffered media. To circumvent these bottlenecks, we have developed a rapid and simple method. We find that Brassica napus can be transformed by dipping directly into an Agrobacterium tumefaciens culture supplemented with surfactant, eliminating the need for media exchange to a buffered solution. We report a method of transformant selection by soaking seeds with antibiotic. These methods save time and money, and reduce the possibility of contamination.
\end{abstract}

Keywords: Brassica napus, floral-dip, Agrobacterium Tumefaciens, Antibiotic, Identify

\section{Introduction}

Transformation of plants usually requires sophisticated regeneration methods that imply technical abilities. The first Arabidopsis transformation method required tissue culture and regeneration(DIRK VALVEKENS 1988); however, in planta transformation protocols have been developed to eliminate these steps (Seok So Chang 1994; Vesna Katavic 1994). Arabidopsis transformation mediated by vacuum infiltration of inflorescences with an Agrobacterium suspension was first introduced by Bechtold et al. (Bechtold N 1993). This method has been widely used in preference to tissue culture techniques as it directly produces transformed seed and negates lengthy and complicated tissue culturing procedures. This transformation 
method was further modified by Clough and Bent(Bent 1998) who demonstrated that the method was just as effective without vacuum infiltration. But the tall plants (such as Brassica napus) were difficult to use this method, then the floral dip method was used. The floral dip method of transformation by immersion of inflorescences in a suspension of Agrobacterium tumefaciens is the method of choice for Brassica napus transformation. These methods allow plant transformation without the need for tissue culture (Bechtold N 2000; Clough SJ 1998), but the growth of the Agrobacterium tumefaciens strain in liquid culture calls for pelleting the culture and resuspending in a buffered media. This typically takes one hour after the time spent preparing the buffered media (Amanda M Davis 2009). Progeny seed are harvested and selection is applied to the resultant seedlings as they germinate. However, Selection for kanamycin- and hygromycin B-resistance in MS medium is a lengthy process. During this lengthy period, fungal contamination may be a problem. Fungal contaminants may be present in seed stocks generated from floral dipping, because sucrose is used in the dipping medium and plants will have been, at least initially, kept in a warm damp environment providing ideal conditions for fungal growth. During seedling selection, fungal contaminants may deplete antibiotic present in the selection medium, such that non-transformants are able to remain green. Crowding of seedlings on an agar plate may allow root growth above the medium and so result in a delay in bleaching of non-transformants due to decreased concentrations of selection agent. To address these problems, we developed a robustly effective in the generation and selection of transgenic Brassica napus first, we provide a description of a bacterial-growth media that supports direct dipping and plant transformation after the trivial addition of surfactant, thereby eliminating the need to exchange bacteria from growth media to a buffer. Second, we provide an improvement method on current selection as it allows quicker identification of transformed seedlings: transformed seedlings are easily discernable from non-transformants this current protocols.

\section{Materials and methods}

\subsection{Plant material and growth condition}

Brassica napus cv. "Ningyou 16" was used as the transformation recipient plant, and sow in experiment farm in October, 2007. Brassica napus cv. "Ningyou 16" rapeseed plant at bud stages were transformed through Agrobacterium-mediated gene transfer method of floral-dip in April, 2008.

\subsection{Agrobacterium strain and plasmids}

Transformations of Brassica napus were performed by the floral dip method (Clough SJ 1998) using Agrobacterium tumefaciens strain LBA4404. The following binary plasmids were used: pCAMBIA2200 which confers kanamycin resistance via the nptII gene and pCAMBIA1300 which confers hygromycin B resistance via the hpt gene. The initial bacterial cultures were grown in $5 \mathrm{ml}$ of LB liquid medium with corresponding antibiotic at $28^{\circ} \mathrm{C}$ and $220 \mathrm{rpm}$, then an inoculum transferred into $500 \mathrm{ml} \mathrm{LB}$ liquid medium at $28^{\circ} \mathrm{C}$ and $220 \mathrm{rpm}$ for two more days (Ming-Hsan Chung 2000).

\subsection{Plant transformation}

The (Main racemes of) rapeseed plants were transformed at bud stages (the initial blossom stage). The plant racemes were submerged in a $20 \mathrm{ml}$ beaker with Agrobacterium medium containing 3\% sucrose, 0.1\% Silwet L-77, 2 ng/1 6-BA and $8 \mathrm{mg} / \mathrm{l}$ acetosyringone (Clough SJ 1998).The inflorescence of rapeseed submerged were covered with sulphate paper bags to keep moist for 24h (Conny Bartholmes 2008).

\subsection{Plant selection}

Seeds were soaked with kanamycin monosulphate solution at a concentration of $300 \mathrm{mg} / \mathrm{l}$ or hygromycin B at a concentration of $100 \mathrm{mg} / 1$, after 24-36 hours incubation, seeds are sown in the soil. After one week, kanamycin-resistant Brassica napus seedlings and kanamycin-susceptible seedlings had significant difference (figure 1).

\subsection{Transgenic detection by PCR}

Genomic DNA was extracted with the CTAB method (Rogers 1988) from leaves of randomly chosen plants transformed with target gene and non-transformed plants as a negative control. PCR analysis was performed using the two primers of target genes. All PCR products were visualized on a 1.0\% agarose gel containing ethidium bromide. The expected length of the amplified product is correct.

\section{Results}

\subsection{Transformation protocol}

The Agrobacterium medium containing Silwet L-77, sucrose, 6-BA and acetosyringone was sufficient for floral-dip transformation. The transformation rates (about 2-3\%, data not shown) reported here were similar to the transformation yield of the traditional floral-dip protocol (Amanda M Davis 2009; Bent 1998; Ming-Hsan Chung 2000). We now consider the need to exchange growth media to a buffered solution to be entirely eliminated.

\subsection{Selection of kanamycin-resistant transformants}

After being soaked in water containing 300mg/l kanamycin, rapeseeds were sown in the soil, When wild-type and 
kanamycin-resistant seedlings growing in the soil were examined after 2 weeks, kanamycin-resistant transformants accumulate chlorophyll and grow photoautotrophically. Transformants had green, open, expanded cotyledons. Non-transformants failed to accumulate chlorophyll; they had pale, closed and unexpanded cotyledons (Figure 2). This selection regime produced green transformants that were easily distinguished from yellow non-transformants in the soil.

To test the reliability of the selection protocol, PCR was applied to detect the candidate transgenic plants. We have confirmed the simple of this rapid selection method with different B. napus. The rapid selection method, as described in the protocol, has been used successfully to identify transformants seedlings (data not shown).

\subsection{Selection of hygromycin-resistant transformants}

Seedlings grown in the presence of hygromycin B displayed a different morphology to those grown in the presence of kanamycin. After 2 weeks in the soil containing $100 \mathrm{mg} / \mathrm{l}$ hygromycin B, hygromycin-resistant transformants had long hypocotyls of approximately 4.5-5.0 cm, whereas non-transformants had short hypocotyls $(2.5-3.0 \mathrm{~cm}$ ) (Figure 3). Additionally, in contrast to seedlings grown on kanamycin, all seedlings grown in the soil containing hygromycin B were green after 2 weeks. However, selection of hygromycin-resistant seedlings from non-transformants was easily achieved by selecting those with elongated hypocotyls.

To confirm the phenotypes of seedlings grown in the presence of hygromycin B, wild-type controls were grown alongside previously characterized hygromycin-resistant lines obtained following transformation with pCAMBIA1300 which confers hygromycin B resistance via the hpt gene. The majority of characterized: hygromycin-resistant seedlings had long hypocotyls following rapid selection. The rapid selection method, as described in the protocol, has been used successfully to identify transformants seedlings (data not shown). PCR analysis was performed using the primers of target genes. All PCR products were visualized on a 1.0\% agarose gel containing ethidium bromide. The expected length of the amplified product is correct (figure4).

\section{Discussion}

We have developed a method that dips and transforms plant directly after the trivial addition of surfactant and distinguishes kanamycin- and hygromycin B-resistant seedlings from non-resistant seedlings through soaking seeds in antibiotics solution. We provide a description of a bacterial-growth media that supports direct dipping and plant transformation after the trivial addition of surfactant, thereby eliminating the need to exchange bacteria from growth media to a buffer. It reduced the risk of seedling loss by pathogen infection. The protocol produces easily identifiable. We also provide an improvement methods on current selection as it allows quicker identification of transformed seedlings: transformed seedlings are easily discernable from non-transformants this current protocols. Kanamycin-resistant seedlings can be distinguished from non-transformant seedlings by the presence of chlorophyll in the expanded cotyledons. However, all seedlings grown in the presence of hygromycin B have green cotyledons, and the selection is based on hypocotyls length. Hygromycin-resistant seedlings have extended hypocotyls in the simple selection method, whereas non-ransformant seedlings have short hypocotyls. The different effects of hygromycin B and kanamycin on hypocotyl elongation may be related to the target of these antibiotics. Hygromycin B inhibits cytosolic protein synthesis (Cabanas MJ 1978), whereas kanamycin inhibit plastid protein synthesis (Gray JC 1984) and glutamine synthase activity (Tachibana K 1986), respectively. This suggests that cytosolic, but not plastid, protein synthesis is required for hypocotyl elongation in the dark. The length of hypocotyls extension in the dark is proportional to the amount of lipid reserve mobilized (Penfield S 2004) and thus remaining reserves may influence the extent of chlorophyll synthesis on transfer of seedlings to the light. non-transformed seedlings grown on hygromycin B have short hypocotyls and are able to synthesize chlorophyll in the light, whereas non-transformed seedlings grown on kanamycin have long hypocotyls and are unable to make chlorophyll (Samuel J Harrison and Cottage 2006).

\section{Acknowledgments}

This work was supported by The National Basic Research Program (973 Program) (2006CB101600) and Talent Fund of Jiangsu University (05JDG003).

\section{References}

Amanda M Davis AH, Andrew J Millar, Chiarina Darrah Seth J Davis. (2009). Protocol: Streamlined sub-protocols for floral-dip transformation and selection of transformants in Arabidopsis thaliana. Plant Methods, 5:3

Bechtold N EJ, Pelletier G. (1993). In planta Agrobacterium-mediated gene transfer by infiltration of adult Arabidopsis thaliana plants. C R Acad Sci Paris. Sciences de la vie/Life Sciences, 316:1194-1199.

Bechtold N EJ, Pelletier G. (2000). In planta Agrobacterium mediated gene transfer by infiltration of adult Arabidopsis thaliana plants. Life Sciences, 316:1194-1199.

Bent SJCaAF. (1998). Floral dip: a simplified method for Agrobacterium-mediated transformation of Arabidopsis thaliana. The Plant Journal, 16(6):735-743. 
Cabanas MJ VD, Modolell J. (1978). Dual interference of hygromycin B with ribosomal translocation and with aminoacyltRNA recognition. Eur J Biochem, 87:21-27.

Clough SJ BA. (1998). Floral dip: a simplified method for Agrobacterium mediated transformation of Arabidopsis thaliana. Plant J., 16:7:35-43.

Conny Bartholmes PN, Günter Theißen. (2008). Germline transformation of Shepherd's purse (Capsella bursa-pastoris) by the 'floral dip' method as a tool for evolutionary and developmental biology. Gene, 409:11-19.

DIRK VALVEKENS MVM, MIEKE VAN LIJSEBETTENS. (1988). Agrobacterium tumefaciens-mediated transformation of Arabidopsis thaliana root explants by using kanamycin selection. Proc Natl Acad Sci USA, 85:5536-5540.

Gray JC PA, Smith AG. (1984). Protein synthesis by chloroplasts In Chloroplast biogenesis, Edited by: Elis RJ Cambridge Univ Press 137-163.

Ming-Hsan Chung M-KC, Shu-Mei Pan. (2000). Floral spray transformation can efficiently generate Arabidopsis transgenic plants. Transgenic Research, 9:471-476.

Ming-Hsan Chung M-KCS-MP. (2000). Floral spray transformation can efficiently generate Arabidopsis transgenic plants. Transgenic Research, 9:471-476.

Penfield S RE, Gilday AD, Graham S, Larson TR, Graham IA. (2004). Reserve mobilization in the Arabidopsis endosperm fuels hypocotyl elongation in the dark, is independent of abscisic acid, and requires PHOSPHOENOLPYRUVATE CARBOXYKINASE1. Plant Cell, 16:2705-2718.

Rogers SU, Bendich, A.J. (1988). Extraction of DNA from plant tissues. Plant Mol Biol Manual, A6:1-10.

Samuel J Harrison EKM, Kate Parsley, Sue Aspinall, John C Gray and, Cottage A. (2006). A rapid and robust method of identifying transformed Arabidopsis thaliana seedlings following floral dip transformation. Plant Methods, 2:19.

Seok So Chang SKP, Byung Chul Kim,Bong Joong Kang, Dal Ung Kim, Hong Gil Nsm. (1994). Stable genetic transformation of Arabidopsis thaliana by Agrobacterium inoculation in planta. The Plant Journal, 5:551-558.

Tachibana K WT, Sekizuwa Y, Takematsu T. (1986). Action mechanism of bialaphos. 2. Accumulation of ammonia in plants treated with bialaphos. $J$ Pest Sci, 11:33-37.

Vesna Katavic GWH, Darwin Reed Marilyn Martin, Ljerka Kunst. (1994). In planta transformation of lirabidopsis thaliana. Mol Gen Genet, 245:363-370.

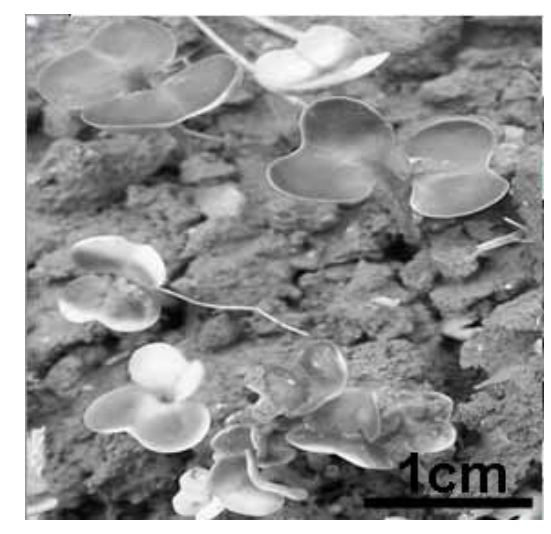

Figure 1. kanamycin-resistant Brassica napus seedlings and kanamycin-susceptible seedlings had significant difference after sowing one week

Transformants had green, open, expanded cotyledons. Non-transformants had pale, closed and unexpanded cotyledons. Size bars $1 \mathrm{~cm}$. 


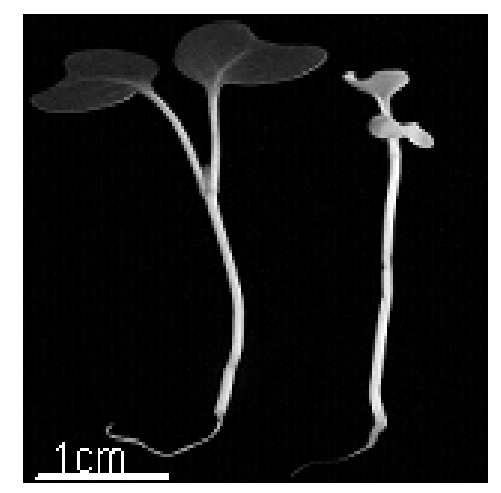

Figure 2. Simple selection of kanamycin-resistance Brassica napus seedlings.

Simple selection of kanamycin-resistant Brassica napus seedling after simple selection procedure(left); kanamycin-susceptible seedling after simple selection procedure (right). Size bars $1 \mathrm{~cm}$.

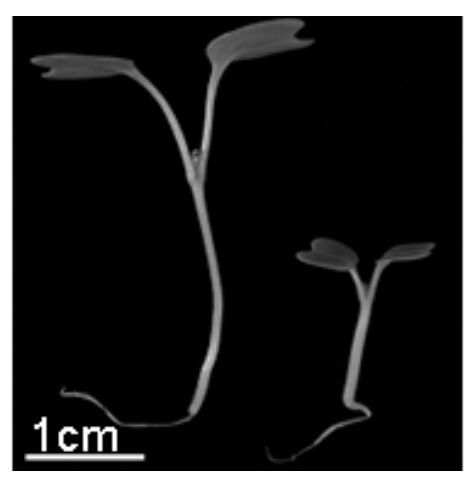

Figure 3. Simple selection of hygromycin-resistance Brassica napus seedlings

Hygromycin-resistant Brassica napus seedling after simple selection procedure (left); hygromycin-susceptible seedling after simple selection procedure (right). Size bars $1 \mathrm{~cm}$.

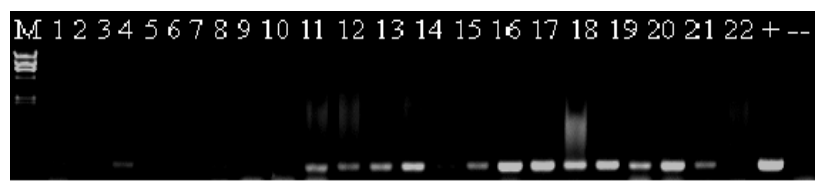

Figure 4. The fragments are amplified by PCR with the primers of target gene from genomic DNA of kanamycin-resistance Brassica napus seedlings and hygromycin-resistance Brassica napus seedlings

The "M" was marker. The lanes 1, 3-6 and 9-22 showed the amplification of the 375 bp DNA fragment of the target gene. The lanes 2, 7 and 8 showed that they were non-transgenic. The lane "+" was PCR product with plasmid as template. The lane "-” was PCR product with genomic DNA of non-transgenic plant as template. 